\title{
Comprehensive Analyses of Genomes, Transcriptomes and Metabolites of Neem Tree
}

Nagesh A Kuravadi, Vijay Yenagi, Kannan Rangiah, HB Mahesh, Anantharamanan Rajamani, Meghna D Shirke, Heikham Russiachand, Ramya Malarini Loganathan, Chandana Shankara Lingu, Shilpa Siddappa, Aishwarya Ramamurthy, Sathyanarayana B N, Malali Gowda

Neem (Azadirachta indica A. Juss) is one of the most versatile tropical evergreen tree species known in India since the Vedic period (1500 BC-600 BC). Neem tree is a rich source of limonoids, having a wide spectrum of activity against insect pests and microbial pathogens. Complex tetranortriterpenoids such as azadirachtin, salanin and nimbin are the major active principles isolated from neem seed. Absolutely nothing is known about the biochemical pathways of these metabolites in neem tree. To identify genes and pathways in neem, we sequenced neem genomes and transcriptomes using next generation sequencing technologies. Assembly of Illumina and 454 sequencing reads resulted in 267 $\mathrm{Mb}$, which accounts for $70 \%$ of estimated size of neem genome. We predicted 44,495 genes in the neem genome, of which 32,278 genes were expressed in neem tissues. Neem genome consists about 32.5\% (87 Mb) of repetitive DNA elements. Neem tree is phylogenetically related to citrus, Citrus sinensis. Comparative analysis anchored $62 \%$ (161 Mb) of assembled neem genomic contigs onto citrus chromomes. Ultrahigh performance liquid chromatography-mass spectrometry-selected reaction monitoring (UHPLC-MS/SRM) method was used to quantify azadirachtin, nimbin, and salanin from neem tissues. Weighted Correlation Network Analysis (WCGNA) of expressed genes and metabolites resulted in identification of possible candidate genes involved in azadirachtin biosynthesis pathway. This study provides genomic, transcriptomic and quantity of top three neem metabolites resource, which will accelerate basic research in neem to understand biochemical pathways. 
2 Comprehensive Analyses of Genomes, Transcriptomes and Metabolites of Neem Tree

3

4 Nagesh Kuravadi A ${ }^{1 \$}$, Vijay Yenagi ${ }^{1 \$}$, Kannan Rangiah ${ }^{2}$, HB Mahesh ${ }^{1,4}$, Anantharamanan Rajamani ${ }^{1}$, 5 Meghna Shirke ${ }^{1}$, Russiachand Heikham ${ }^{1}$, Ramya Malarini L1, Chandana Shankara Lingu ${ }^{1}$, Shilpa

6 Siddappa $^{1}$, Aishwarya Ramamurthy ${ }^{1}$, Sathyanarayana B N ${ }^{3}$ and Malali Gowda ${ }^{*}$

81 Genomics Laboratory, Centre for Cellular and Molecular Platforms, National Centre for Biological

9 Sciences, Bangalore 560065

102 Metabolomics Facility, Centre for Cellular and Molecular Platforms, National Centre for Biological

11 Sciences, Bangalore 560065

123 Plant Tissue Culture Laboratory, University of Agricultural Sciences, GKVK, Bangalore 560065

134 Marker Assisted Selection Laboratory, Department of Genetics and Plant Breeding, University of

14 Agricultural Sciences, GKVK, Bangalore 560065

$15 \$$ - Authors contributed equally to the work

$16 *$ - To whom correspondence should be addressed.

17 E-mail: malalig@ncbs.res.in

20 Abstract

21 Neem (Azadirachta indica A. Juss) is one of the most versatile tropical evergreen tree species known in 22 India since the Vedic period (1500 BC-600 BC). Neem tree is a rich source of limonoids, having a 23 wide spectrum of activity against insect pests and microbial pathogens. Complex tetranortriterpenoids 24 such as azadirachtin, salanin and nimbin are the major active principles isolated from neem seed. 25 Absolutely nothing is known about the biochemical pathways of these metabolites in neem tree. To 26 identify genes and pathways in neem, we sequenced neem genomes and transcriptomes using next 27 generation sequencing technologies. Assembly of Illumina and 454 sequencing reads resulted in 267 $28 \mathrm{Mb}$, which accounts for $70 \%$ of estimated size of neem genome. We predicted 44,495 genes in the 
29 neem genome, of which 32,278 genes were expressed in neem tissues. Neem genome consists about $3032.5 \%(87 \mathrm{Mb})$ of repetitive DNA elements. Neem tree is phylogenetically related to citrus, Citrus 31 sinensis. Comparative analysis anchored $62 \%(161 \mathrm{Mb})$ of assembled neem genomic contigs onto 32 citrus chromosomes. Ultrahigh performance liquid chromatography-mass spectrometry-selected 33 reaction monitoring (UHPLC-MS/SRM) method was used to quantify azadirachtin, nimbin, and 34 salanin from neem tissues. Weighted Correlation Network Analysis (WCGNA) of expressed genes and

5

KEY words: $A$. indica, azadirachtin, next generation sequencing, annotation

Abbreviations: $\mathrm{nts}=$ Nucleotides, UHPLC-MS/SRM $=$ Ultrahigh performance liquid chromatography-mass spectrometry-selected reaction monitoring, $\mathrm{Kb}=$ Kilobases, $\mathrm{RPKM}=$ reads per kilo base per million, WGCNA $=$ Weighted Correlation Network Analysis, SNP $=$ Single nucleotide polymorphism, SSR $=$ Simple sequence repeat.

\section{Introduction}

Neem, Azadirachta indica is an evergreen tree, native to the Indian subcontinent. It belongs to Meliaceae family plants, which are the major source for diverse limonoids (Tan \& Luo 2011). Neem has been used in Ayurveda, Siddha, Unani and other Indian local health traditions. Over 700 herbal preparations and over 160 local practices containing neem ingredients are known in India, which claim to prevent various ailments or disorders in humans (Brahmachari 2004). Neem based pesticidal 52 formulations are widely regarded as organic, and are found to have low toxicity against non-target 3 beneficial organisms as compared to synthetic pesticides. Nimbin was the first chemical limonoid 4 isolated from neem tree (Siddiqui 1942). Subsequently, more than 150 bioactive chemical compounds 55 have been isolated from various neem tissues (Brahmachari 2004). 
56 Azadirachtin is the major tetranortriterpenoid in neem seeds (Butterworth \& Morgan 1968) and its 57 molecular structure elucidation took more than 20 years of research (Broughton et al. 1986). 58 Azadirachtin is one of the highly successful bio pesticides in the world, which is isolated from neem 59 seeds and is non-persistent in the environment. Its content is highly variable in trees in various 60 locations due to genetic variability and environmental factors (Sidhu et al. 2003). Chemical in-vitro 61 synthesis of azadirachtin has been tried in the laboratory. However, long synthesis process and 62 molecular complexity was realized and that chemical synthesis of azadirachtin is not a viable method 63 for commercial production (Veitch et al. 2008). Hence, there is an increased interest to understand the

73 Materials and Methods

74 Neem genotypes

75 Individual neem tree was identified from three varied geographical regions of southern India including 76 Karnataka [GKVK, Bangalore (abbreviated as Genotype 1), Anuganalu, Hassan (Genotype 2)] and 77 Tamil Nadu (Erode; Genotype 3) for this study (Supplementary Figure 1).

\section{Isolation of genomic DNA and total RNA from neem tissues}

80 Mature neem leaves were collected from neem trees for DNA isolation. CTAB method (6\% CTAB, $811.4 \mathrm{M} \mathrm{NaCl}, 20 \mathrm{mM}$ EDTA, $10 \mathrm{mM}$ Tris Base pH8) was used for isolation of neem genomic DNA 82 (Doyle 1990), with few modifications. Neem DNA isolation is a difficult process due to the presence 
83 of highly oxidized complex compounds in neem leaves. Instead of precipitating the DNA using iso-

84 propanol as in CTAB method, we used the supernatant to purify genomic DNA using the Sigma 85 Genelute plant DNA isolation kit (G2N70, Sigma). RNA isolation was carried out using $100 \mathrm{mg}$ of the 86 neem tissues using Sigma Spectrum Plant Total RNA Kit (STRN50, Sigma). DNase treatment was 87 done using DNASE digestion kit (DNASE 70, Sigma). DNA free RNA was dissolved in DEPC water. 88 The RNA integrity (RIN) was determined using Agilent Bioanalyzer.

\section{Induction of callus tissue}

91 Neem endosperm explants, of 4-5mm length pieces were cultured on Murashige and Skoog solid 92 medium ( $\mathrm{pH}$ 5.6) containing 0.45mg BAP, 0.80mg NAA and 150mg Casein hydrolysate. Cultures 93 were maintained in growth chamber at $25 \pm 2{ }^{\circ} \mathrm{C}$ with $55 \pm 5 \%$ of relative humidity, for $16 \mathrm{~h}$ 94 photoperiod and allowed for formation of callus tissue. After 2 weeks, mature callus was sub-cultured 95 to fresh media every fourth or sixth weeks.

\section{NGS library construction and sequencing}

98 Whole genome shotgun DNA library was prepared using Illumina TrueSeq DNA sample preparation 99 kit (FC-121-2001). The paired-end (PE) (2x100 nts) sequencing was carried out using Illumina HiSeq

1001000 at the Next Generation Genomics Facility at Centre for Cellular and Molecular Platforms (C101 CAMP). We prepared whole genome shotgun 454 library for Genotype 1 (GKVK, Bangalore) using 102 the rapid library preparation kit from Roche (Cat. No. 05608228001y; version 4.0.12). The 454 103 sequencing was carried out using GS FLX+ chemistry as per Roche/454 manual instructions 104 (http://454.com).

\section{Transcriptome library preparation and sequencing}

107 Total RNA isolation was carried out for $100 \mathrm{mg}$ of the neem tissues using the Sigma Spectrum Plant 108 Total RNA Kit (STRN50, Sigma). The RNA-seq library was prepared using $1 \mu \mathrm{g}$ of total RNA 109 according to Illumina's TruSeq RNA sample preparation kit (RS-122-2001). 


\section{De novo whole genome assembly}

112 Illumina PE reads were pre-processed using FASTX-Toolkit (v 0.0.13). The read quality score cut-off

113 (q) and percentage (p) value was assigned as 20 and 100, respectively (i.e. $q / 20$ and p/100). After

114 quality filtering, we obtained a total 75-192 millions of paired-end reads and 61-71 millions of

115 singleton reads (Supplementary Table 1). De novo assembly of neem genome was performed using

116 Velvet program (Zerbino \& Birney 2008). We optimized the Velvet assembly through iteratively

117 process for various k-mers (27 to $67 \mathrm{nts}$ ). The Velvet assemblies with best k-mers in the size of 45,45

118 and 33 were used for Genotypes 1, 2 and 3, respectively. The parameter used for deciding the best k-

119 mer for theoretical coverage were N50, maximum contigs length, totals contigs, assembled genome 120 size and total number of reads used (Supplementary Table 1).

121454 reads were assembled using MIRA software (Chevreux 2005). The first step of the 122 assembler is to compare every read with every other read (and its reversed complement) to detect 123 potential overlaps. These potential overlaps were examined with Smith-Waterman-based algorithm for 124 local alignment of overlaps. If overlaps were found, then they were verified using Smith-Waterman 125 methods and are assembled into contigs. The software used default parameters (minimum read length 126 of $40 \mathrm{nts}$ and minimum base quality of q10) with single end read format.

128 Hybrid neem genome assembly generation using Illumina and 454 contigs

129 Hybrid assembly of neem genome was carried out by merging contigs from Illumina and 454 sequence 130 reads. The Genotype 1 (GKVK, Bangalore) assembled contigs, obtained from Velvet (Zerbino \& 131 Birney 2008) and MIRA (Chevreux 2005) assemblers were merged using clustering program, CD-HIT132 est by keeping minimum similarity cut-off of 90\% ( $\mathrm{Li} \&$ Godzik 2006). This clustering approach

133 allowed both Illumina and 454 contigs to merge and build longer contigs of the genome. A total of 13494,780 Illumina contigs were clustered with a total of 121,184,454 contigs, resulting in a total of 13568,604 unique contig sequences (Table 1). 


\section{Eukaryotic core gene mapping}

138 The completeness of the assembled genome was checked by using eukaryotic core gene-mapping 139 approach (CEGMA) (Parra et al. 2007). CEGMA used 248 core eukaryotic genes that are highly 140 conserved and single-copy genes in eukaryotic genomes.

142 Nuclear gene prediction and annotation

143 Genes were predicted from hybrid genome assembly using Augustus (Stanke et al. 2006) and GenScan 144 (Burge \& Karlin 1997) (Supplementary Table 2). Then we clustered genes with similarity cut-off 90\% 145 using CD-HIT-est program (Weizhong Li \& Adam Godzik 2006). This method gave the overall 146 representation of genes, by merging similar genes predicted by both the programs. Then we discarded 147 genes that were less than $100 \mathrm{bp}$ in size (Delcher et al. 1999). Then BLAT was used to obtain the 148 number of unique and common genes from the CD-HIT out file (Kent 2002). Genes originated from 149 repeat elements were identified using Repeat Modeler program

150 (http://www.repeatmasker.org/RepeatModeler.html). Gene expression was quantified by mapping 151 RNA-Seq reads (Table 2). We used all the genes with and without RNA-seq evidence to search gene 152 functions using UniProt database, Gene Ontology (GO), Kyoto Encyclopedia of Genes and Genomes

153 (KEGG) and Enzyme Commission number (EC). Schematic representations of GO classes in neem

154 genome are summarized in Supplementary Figure 2. Above analyses was done using BLASTX 155 (Altschul et al. 1990) in annot8r (Schmid \& Blaxter 2008) with E-value cut-off of 10-3. Genes with 156 multiple hits were filtered based on E-value; the annotation details for each gene are listed in the 157 Supplementary Table 3.

\section{Gene expression analysis}

160 The combined transcriptome of twelve tissues of neem tree from Genotype 1 were assembled using

161 Trinity software (Grabherr et al. 2011). The transcripts were clustered to remove the over represented 162 short fragments using CD-HIT-est program (Li \& Godzik 2006) with a minimum similarity cut-off of $16390 \%$. The 44,495 genes from CD-HIT-est were mapped with RNA-seq data from individual tissues 
164 using SeqMap (Jiang \& Wong 2008). The mapping of RNA-seq reads from each tissue were used to 165 measure the expression value in RPKM (reads per kilo base per million) using rSeq tool (Jiang \& 166 Wong 2009). The gene expression was estimated using RPKM value minimum $\geq 1$ for further analysis.

167 The RPKM value was used to cluster the genes according to their expression pattern using WCGNA 168 package in $\mathrm{R}$ tool (Langfelder \& Horvath 2008). The expression value was also determined for 169 assembled transcripts to verify expression of genes predicted from gene models.

\section{Repeat prediction from neem genomes}

172 De novo repeat identification was done using RepeatModeler

173 (http://www.repeatmasker.org/RepeatModeler.html). The program was run with RM-BLAST (NCBI)

174 database as an input for the repeat modelling. We trained Repeat Modeler using genomes of other 175 published plant species including P. trichocarpa (39.40\%), R. communis $(51.74 \%)$ and A. thaliana 176 (15.29\%) for pipeline validation (Supplementary Table 4). We downloaded NCBI data (SRA1085705) 177 from Krishnan et al (2012) and re-built the neem genome assembly using SOAPdenovo2 program with 178 different k-mers (Supplementary Table 5).

\section{Quantitative PCR (qPCR) analysis}

181 Total RNA from leaf, callus and developing endosperm ( $\mathrm{S} 1=10$ days post seed setting, $\mathrm{S} 2=20$ days 182 post seed setting, $\mathrm{S} 3=30$ days post seed setting, $\mathrm{S} 4=40$ days post seed setting) was isolated using 183 TRIzol reagent method and quantified using Qubit Fluorometer. The cDNA synthesis was performed 184 using total RNA $(1 \mu \mathrm{g})$ with oligo $(\mathrm{dT})$ random primers $(50 \mu \mathrm{M})$ and SuperScript ${ }^{\circledR}$ III RT enzyme 185 (200u/ $\mu$ l) (Life Technologies, Cat \# 18080044). The qPCR was performed on Applied Biosystems, 186 7900HT Fast Real-Time PCR system machine. Real-time PCR was performed in a 384-wells optical 187 reaction plate (Applied Biosystems, Foster City, USA) using SYBR green PCR mastermix (Life 188 Technologies, Cat \# 4344463), which contains AmpliTaq Gold ${ }^{\circledR}$ DNA polymerase and ROX as a 189 passive reference dye. Cycling conditions were $95^{\circ} \mathrm{C}$ for $15 \mathrm{sec}, 60^{\circ} \mathrm{C}$ for $30 \mathrm{sec}$ and $72^{\circ} \mathrm{C}$ for $30 \mathrm{sec}$ 190 with 40 cycles. We compared the fold change in qPCR experiments for selected genes from 
191 azadirachtin biosynthesis with conserved eukaryotic (rice) elongation factor $1 \alpha$ (eEF-1 $\alpha$ ) gene 192 (AK061464.1). All reactions were performed in triplicate with elongation factor primers and water as 193 an internal control. The primer sequences of selected genes are listed in the Supplementary Table 6.

194

195 SSRs, SNPs and InDels analysis

196 Identification of simple sequence repeats (SSRs) or microsatellite was done using MIcroSAtellite tool 197 (MISA) (http://pgrc.ipk-gatersleben.de/misa/) with assembled neem genome sequences. The SSRs 198 containing contigs were extracted for SSRs motif variability prediction. The previously published SSR 199 marker regions (Boontong et al. 2009) for neem were compared to the newly identified SSRs from the 200 genome. The SSR based polymorphism among the neem accessions was done using an in-house 201 software pipeline. This pipeline uses the identified SSR region along with 100 bp upstream and 202 downstream from each SSR loci. The SSR regions were aligned to each other for a pair of genomes 203 using Bowtie2 alignment. The resulting .SAM files were parsed using the libraries function of genomic 204 ranges (Aboyoun et al. 2010), Gtools (Warnes et al. 2008) and Stringr (Wickham 2010) in R program 205 to obtain polymorphic SSR regions between neem genomes. The concordance was taken with neem 206 tree Genotype 1 as a reference to shortlist the most polymorphic SSRs.

207 Single nucleotide polymorphism (SNP) and Insertion Deletion (InDels) markers were identified by 208 mapping Illumina short reads from Genotype 2 and Genotype 3 to reference Genotype 1 assembly 209 using Bowtie2 (Langmead \& Salzberg 2012). The alignment results were converted into .BAM format 210 using Samtools v1.19 (Li et al. 2009). All the .BAM files were merged into a combined .BAM file. The 211 .BAM file was sorted, indexed and duplicates removed using Samtools v1.19 for further analyses. VCF 212 file was generated for each genome to obtain SNPs and InDels from neem genome. The SNPs and 213 InDels were further filtered to obtain aligned reads with quality $>30$, and minimum sequence depth of 21410 reads. The SNPs were annotated using snpEff tool (Cingolani et al. 2012). 215

\section{Neem chloroplast and mitochondrial genome assembly and annotation}


217 The reads for chloroplast and mitochondria were extracted separately by mapping genome reads to 218 chloroplast and mitochondrial genomes of known plants; A. thaliana, B. napus, C. papaya, C. sinensis, 219 N. tabacum, P. dactylifera, P. trichocarpa, R. communis, S. bicolour and V. vinifera using Bowtie2 220 (Langmead \& Salzberg 2012). The mapped reads were extracted using Samtools (Li et al. 2009) and 221 assembled separately using Velvet (Zerbino \& Birney 2008). Gene prediction and assembly of 222 chloroplast genome was done using DOGMA (Wyman et al. 2004). Gene prediction and annotation of 223 the mitochondrial assembly was done using Mitofy (Alverson et al. 2010).

\section{Synteny analysis}

226 We aligned neem contigs on citrus (Citrus sinensis) chromosomes using MUMMER program (Kurtz et 227 al. 2004). Synteny was computed among neem and citrus using Symap4.0 (Soderlund et al. 2011). The synteny information was visualized using the Perl script provided with Symap4.0.

\section{Classification of gene families and phylogenetic analysis}

231 The proteome of 23 sequenced plant species along with neem were used to search for homologues and 232 unique genes. All-vs-all BLAST-P (E-value e-10) was done using Proteinortho program (Lechner et al. 233 2011). Comparative data from BLAST analysis was further classified into list of potential orthologs, 234 co-orthologs and paralogs. Besides classifying, the program has also grouped the proteins into specific 235 groups by clustering the gene-pairs. The gene content at the ancestral nodes along with the branches 236 was reconstructed by using Wagner Parsimony and Likelihood-based approaches in the program count 237 (Csüös 2010).

\section{Quantification of azadirachtin, salanin and nimbin using UHPLC-MS/SRM method}

240 We purchased neem standard metabolites such as azadirachtin (Cat. No. A7430 from Sigma-Aldrich, 241 India), salanin (Cat. No. ASB-00019028-005 from Chromodex, USA) and nimbin (Cat. No. N476280 
242 from Toronto Research Chemicals, Canada). High purity MS grade solvents (methanol, acetonitrile and 243 water) were obtained from Merck Millipore (Merck Millipore India Pvt. Ltd., Mumbai).

244 For metabolites analysis, we collected samples from various neem tissues (mature fruit, developing 245 endosperm, mature leaf, flower and bud, fruit coat and pulp and seedling shoot and root) and dried at $24637 \mathrm{oC}$ for two days. The samples were ground into fine powder using Pestle and Mortar, and stored at $24780 \mathrm{oC}$ until use. There were no commercially internal standards available for neem metabolites 248 quantification; therefore, we used estrone-d4 (Steraloids Inc, USA) as an internal standard to construct 249 the standard curve and also for absolute quantification. The calibration curves for the quantification of 250 all three neem metabolites were linear over a 64-fold concentration range (azadirachtin and salanin) 251 and 73-fold concentration range (nimbin) with linear regression correlation coefficients ranging from 2520.998 to 0.999 (Figure 1D). Typical UHPLC-MS/SRM chromatogram profile for neem metabolites 253 from standard compounds and from the seed extract are shown in Figure 1C. All analytes showed 254 single sharp peak in C-18 column.

255 Neem metabolites were extracted from the dried powder $(2 \mathrm{mg})$ using $1 \mathrm{~mL}$ of methanol followed by 5 $256 \min$ sonication and centrifuged for $5 \min \left(13000 \mathrm{rpm}, 10^{\circ} \mathrm{C}\right)$, the supernatant was transferred to fresh 257 micro-centrifuge tubes. About $5 \mu \mathrm{L}$ of supernatant was spiked into $35 \mu \mathrm{L}$ of methanol along with 258 internal standard $(10 \mu \mathrm{L}$ estrone-d4 from $100 \mu \mathrm{g} / \mathrm{mL})$. The analyses were done by injecting $10 \mu \mathrm{L}$ of 259 the sample into the UHPLC-MS/SRM system (LC-Agilent 1290 infinity series, MS - Thermo Fishers 260 TSQ vantage). The intense product ions were selected for the LC-MS/SRM analysis [azadirachtin (703 $261 \rightarrow 567 \mathrm{Da})$, nimbin $(541 \rightarrow 509 \mathrm{Da})$, salanin $(597 \rightarrow 419 \mathrm{Da})$, and for estrone-d4 $(275 \rightarrow 257 \mathrm{Da})]$ 262 We used following LC conditions: solvent system A-Water (10 mM Ammonium acetate) containing $2630.1 \%$ FA and B-Acetonitrile containing 0.1\% FA, Flow-200 $\mu \mathrm{L} / \mathrm{min}$, Column- C-18 column (Shim264 pack, ODS III, $2.1 \times 150 \mathrm{~mm}, 2 \mu \mathrm{m}$ ), Gradient- 2\% B at $0 \mathrm{~min}, 2 \%$ B at $3 \mathrm{~min}, 40 \% \mathrm{~B}$ at $10 \mathrm{~min}, 95 \%$ $265 \mathrm{~B}$ at $15 \mathrm{~min}, 2 \% \mathrm{~B}$ at $15.1 \mathrm{~min}, 2 \% \mathrm{~B}$ at 15.1-20 min. MS conditions: spray voltage, 3000V; ion 266 transfer capillary temperature, $270^{\circ} \mathrm{C}$; source temperature $100^{\circ} \mathrm{C}$; sheath gas 18 , auxillary gas 5 267 (arbitrary units); collision gas, argon; S-lens Voltage was optimized for individual metabolites; scan 268 time of 50 millisec/transition and ion polarity positive. In case of azadirachtin (15.6 pg to $1 \mathrm{ng}$ ), nimbin 
269 (3.4 pg to $0.25 \mathrm{ng}$ ) and salanin ( $7.8 \mathrm{pg}$ to $0.5 \mathrm{ng}$ ) on column was used to construct the standard curve.

270 The overall scheme for the quantification of neem metabolites is shown in the Figure 1. The UHPLC-

271 MS/SRM chromatogram for lowest standard and metabolites from seed, standard curve for three 272 metabolites and final quantification of neem metabolites from various tissues are illustrated. The final 273 absolute quantification was done based on the constructed standard curve (ratio versus concentration) 274 of individual metabolites.

\section{Metabolites pathways analysis}

277 The quantified metabolites data points from different explants or tissues were used to compare the gene 278 expression pattern using WGCNA package in R (Langfelder \& Horvath 2008) for tracking genes 279 involved in azadirachtin biosynthetic pathway. The clustering program in WGCNA provided the initial 280 correlation with gene expression and metabolite concentrations in different tissues of neem tree (Figure 281 2b) (Supplementary Table 7). The annotation of genes from KEGG and BLAST were used to select the 282 candidate genes in secondary metabolite biosynthetic pathway. We selected the genes giving best bit score for each step in terpenoid biosynthetic pathway. The selected gene annotation was confirmed by BLAST results against NCBI-nr database. In case of multiple hit for pathway function, the expression 285 levels of genes in different tissues were compared to match the metabolite concentration by 286 considering developing endosperm and mature leaf as contrasting datasets for azadirachtin concentration. Pearson's correlation value was also calculated between the expression values of each gene in tissues versus Azadirachtin concentration (Supplementary Table 8a). Further genes involved in conversion of squaline to azadirachtin could not be assigned to specified function. However, the genes showing a high expression level in developing endosperm and having high Pearson's correlation with azadirachtin concentration were hypothesized to be part of azadirachtin biosynthesis. The hypothesized set of annotated genes in each pathway is listed in Supplementary Table 8 and also shown in heatmap pattern (Figure 3) using R statistical program. 


\section{Results}

297

298

299

300

301

302

303

304

305

306

307

308

309

310

311

312

313

314

315

316

317

318

319 Gene prediction was performed using two programs, Augustus and GenScan, which led to

320 identification of 40130 and 52617 genes, respectively. In total, we identified 44,495 genes after

321 merging and clustering of genes from these two programs. The genic region of neem genome was 322

\section{De novo sequencing and assembly of neem genome}

We sequenced neem genome using Illumina and 454 platforms. Illumina HiSeq paired-end (2x100 nts) sequencing yielded $13.86 \mathrm{~Gb}$ of high quality data for the neem Genotype 1 (GKVK, Bangalore). De novo assembly resulted in $216 \mathrm{Mb}$ (version 1.1) with $21 \mathrm{X}$ coverage using Velvet software. This analysis produced a total of 94,780 scaffolds where $90 \%$ of the genome was covered by scaffolds length longer than $1000 \mathrm{bp}$. The N50 was about $22 \mathrm{~Kb}$ with $31.13 \%$ of GC content and the longest scaffold length was $241 \mathrm{~Kb}$. In addition to Illumina data, we also generated $1.13 \mathrm{~Gb}(2,762,254$ reads $)$ data using Roche 454 GS FLX + chemistry for the Genotype 1. The average read length was 410 nts and longest 454 read length was 1596 nts. The 454 assembly was generated using MIRA program (Table 1). The draft genome quality was further improved through hybrid assembly by merging Illumina contigs and 454 contigs using CD-HIT-est by keeping minimum similarity cut-off of $90 \%$.

The total size of hybrid genome assembly for neem Genotype 1 was improved to $267 \mathrm{Mb}$. Assembly statistics of the improved assembly neem Genotype 1 is shown in Table 1. Clustering approach in hybrid assembly has significantly reduced the number of contigs from 94,780 to 68,604 and decreased the number of N's from $2.22 \%(4,841,912 \mathrm{nts})$ to $1.81 \%(4,842,395 \mathrm{nts})$. Although clustering approach reduced the $\mathrm{N} 50$ from $22.3 \mathrm{~Kb}$ to $15.95 \mathrm{~Kb}$, the longest and shortest contig length remained unchanged. Hence, the clustered hybrid assembly (version 2.1) was chosen for further detailed analyses. To confirm the completeness of neem genome assembly, we analyzed conserved eukaryotic genes (CEG) in the neem hybrid genome assembly. This analysis was able to identify 224 out of 248 complete CEGs in the neem assembly.

\section{Neem genome annotation} about $114 \mathrm{Mb}$ (42.5\%). The initial gene prediction was carried out without masking the repeat regions 
323 in the genome to avoid missing of simple sequence repeats in the coding regions and to predict proper

324 exon/intron boundaries. To annotate the neem genes, annot8r program was used by incorporating 325 functional proteins from GO, EC and KEGG and merged with BLAST results (Supplementary Table 326 9). There were 29,050 unique genes in the neem genome that were free from any repeat elements.

327 We used RNAseq data to validate predicted neem genes. This analysis revealed thousands of genes 328 expressed in various neem tissues including flower (21,927 genes), mature fruit $(17,366)$, developing 329 endosperm $(19,480)$, mature leaf $(19,308)$, fruit coat and pulp $(21,537)$, seedling root $(20,798)$, and 330 seedling shoot $(20,199)$. The analysis also showed that 3,008 genes exhibited tissue specific expression 331 profile, while 13,711 genes were expressed in all the tissues (Figure 2a). We identified 80,867 332 transcripts $(53 \mathrm{Mb})$ from de novo assembly of neem transcriptome using Trinity program. These 333 transcripts were further used as supporting expression evidences for the genes that involved in 334 metabolites biosynthesis pathways. The protein sequences for 44,495 genes were compared with 335 proteome of 23 sequenced plant species. Of these, 23,125 genes (52\%) were classified into 18,327 336 families (Figure 4). Neem genome found to have 4,320 multi-gene families (Supplementary Table 9).

\section{Gene ortholog analysis}

339 Ancestral orthogroup reconstruction was done using Wagner Parsimony (Supplementary Table10) and 340 likelihood based on birth-death model with equal gain-loss penalty (Supplementary Table 11). The 341 orthogroup reconstruction shows that 5,122 genes gained and 2,755 genes lost in comparison with 342 ancestral nodes based on the gene family expansion in the neem genome.

343 Along with gene classification to orthogroups, the BLAST results of proteinortho (Lechner et al. 2011) 344 showed 24,216 genes (54.42\%) as common between neem and citrus (Figure 5a). The comparative 345 analyses revealed 20279 and 21931 genes that are unique to neem and citrus, respectively. Out of 34620,279 unique genes, 5,832 genes were expressed in various neem tissues.

347 Neem orthologous genes were compared with dicot (Arabidopsis, Populus and grapes) and monocot 348 (rice) plants (Figure 5b). 27,498 genes out of 44,495 genes were found to have orthologs (with more 349 than $60 \%$ identity) in other plant species. Interestingly, $38 \%(16,997)$ of genes from neem did not show 
350 any orthologs in sequenced plant genomes. This analysis identified 16,997 genes that are unique to 351 neem, which support the presence of unique gene families in neem tree. Among these unique genes, 352680 genes share minor homology with hypothetical/predicted proteins in other plant species, while 3532343 were genes found to have no sequence similarity to either predicted or known genes. The list of 354 unique genes in neem is summarized in the Supplementary Table 12. The unique genes were further 355 filtered for presence of repeat elements and expression evidences. More than 3,000 unique genes that 356 have no repeat elements in the neem genes have expression evidence. This indicates that neem repeat 357 derived genes are active in various neem tissues, which are interesting for future studies.

\section{Repeat content in neem tree genome}

360 De novo repeat identification in neem genome was performed using RepeatModeler (Smit \& Hubley 361 2008). This analysis identified $32.53 \%(87 \mathrm{Mb})$ of neem genome constitute for repeat elements, of 362 which $17.06 \%$ of repeats were not annotated to any known repeat families (Table 3). The long terminal 363 repeat (LTR), retro-transposons are the major classes of known repeats, which constitute about $10 \%$ of 364 repeats the neem genome. The detailed repeat prediction is shown in Supplementary Table 13. Our 365 repeat prediction in neem $(32.53 \%)$ is comparable to rice genome. Our RepeatModeler accuracy for 366 repeat prediction in neem genome was further confirmed by independent analyses of the other two re367 sequenced neem genomes (Genotype $2=27.63 \%$, Genotype $3=26.76 \%$ ). To verify repeats in the 368 recently reported neem genome by Krishnan et al (2012), we re-built the neem genome assembly 369 reported by Krishnan et al short read data (SRA:1085705) using SOAPdenovo2 program. During our re-analysis of neem genome assembly from Krishnan et al dataset (SRA1085705), we found 22 to $35 \%$

371 of genome harbour repeats (Supplementary Table 5). In addition, we identified higher gaps with Ns 372 (14.7\% to 60\%) for 31 to $37 \mathrm{k}$-mers from SRA1085705 (Supplementary Table 5) as compared to neem 373 genome assembly (1.81\%) from our study.

\section{DNA polymorphism among neem genotypes}


376 Simple sequence repeats (SSR), single-nucleotide polymorphisms (SNPs) and small insertions and 377 deletions (InDels) are the most abundant DNA markers in any plant genomes. Three sequenced neem 378 genotypes were compared to assess genome-wide genetic diversity. This analysis identified 140807, 379108020 and 95840 SSRs from neem Genotype 1, Genotype 2 and Genotype 3, respectively 380 (Supplementary Table 14a and 14b). 2217, 1665 and 1606 genes were associated with the presence of 381 SSRs motif in neem Genotype 1, 2 and 3, respectively. SSR markers in the genic regions of Genotype 3821 are summarized in Supplementary Table 14a and 14b. Tri-repeats were highest (1,841 SSRs) for 383 genes as compared to other repeat units. The AAG/CTT (533), AG/CT (114) and A/T (127) were the 384 most predominant SSR motifs in genic regions of neem genome (Supplementary Table 14b). The list 385 of SSR associated genes in Genotype 1 has been provided in Supplementary Table 15.

386 With the availability of three neem genomes, we developed an in-house analysis pipeline to predict in-

387 silico polymorphism in SSR loci. Nearly 100 nts from upstream and downstream regions of SSR motif 388 from Genotype 1 (reference) were used for SSR polymorphism analysis. These analyses resulted in 3892,199 and 2,120 polymorphic SSRs in the Genotype 2 and Genotype 3, respectively. The concordance 390 SSR analyses showed 571 SSRs were polymorphic among three neem genomes (Supplementary Table 391 16). The in-silico identified polymorphic SSR markers can be used to test polymorphism in neem 392 germplasm or natural plantation.

394 In addition, SNP and InDel were further analyzed among three neem genotypes using Genotype 1 as a 395 reference genome. This analysis yielded 698173 SNPs and 53508 InDels for Genotype 2 and 860215 396 SNPs and 66171 InDels for Genotype 3, respectively. SNP annotation was carried out to discern 397 presence of SNPs in the coding region of the genome. The SNP annotation showed that $80.70 \%$ and $39879.16 \%$ of SNPs in Genotype 2 and 3, respectively, are located in the non-coding regions (upstream 399 and downstream) of the genes. We identified 130668 (2.78\%) and 159375 (3.21\%) SNPs, for exonic 400 regions of coding genes from Genotype 2 and 3, respectively. There were 31,312 and 37,864 SNPs in 401 the coding sequence which represents the synonymous amino acid changes in protein coding regions of 402 Genotype 2 and 3, respectively. There were 84194 and 102663 SNPs that showed non-synonymous 
403 amino acid substitutions in the protein coding regions of Genotype 2 and 3, respectively. The presence 404 of SNPs in start and stop codons were less as expected. The details of SNP annotation is summarized in 405 Supplementary Table 17.

406

407 Azadirachtin biosynthesis pathways

408 We have quantified neem metabolites using high sensitive UHPLC-MS/SRM method, which can detect 409 the picogram (pg) level of neem metabolites (azadirachtin -15.6 pg, nimbin - 3.4 pg and salanin - 7.8 $410 \mathrm{pg})$. The concentration of neem metabolites in mature seeds [azadirachtin (11046 pg/ $\mu \mathrm{g})$, nimbin (3607 $411 \mathrm{pg} / \mu \mathrm{g})$ and salanin $(5235 \mathrm{pg} / \mu \mathrm{g})]$ was higher than other neem tissues. The concentration of 412 Azadirachtin was always higher in mature seeds, followed by developing endosperm, shoot, root, 413 cambium, pulp, flower, bark and leaf. Nimbin concentration was higher in seed, followed by bark, 414 cambium, endosperm, root, shoot, flower, pulp and leaf. Similarly for salanin, the trend was higher in 415 seeds followed by endosperm, bark, cambium, root, shoot, leaf, pulp and flower. The quantification of 416 neem metabolites from various tissues is shown in Figure 1E. Three of these metabolites are always 417 found to be higher in seeds than other tissues, therefore, we quantified these across various seed 418 developmental stages ( $\mathrm{S} 1=10$ days post seed setting, $\mathrm{S} 2, \mathrm{~S} 3$ and $\mathrm{S} 4=40$ days post seed setting). The 419 level of all these three metabolites was higher across developing seed stages (Figure 6B).

420 Genes involved in various steps from tirucallol to azadirachtin are not yet established. Therefore, we 421 devised bioinformatics approach by clustering expressed genes along with amount of metabolites 422 (azadirachtin, nimbin and salanin) in each tissue using Weighted Correlation Network Analysis 423 (WGCNA) method. This WGCNA clustering analysis identified azadirachtin biosynthesis genes that 424 are up-regulated in developing endosperm, and had a minimal expression in other tissues such as leaf, 425 flower, fruit coat and pulp (Supplementary Table 8a). From this analysis, we identified more than 150 426 genes that were highly expressed in developing endosperm. However, the majority of these genes have 427 homology to other plant species with a Pearson's co-relation value above +0.8 for azadirachtin. To 428 validate our method, we selected 10 genes with high correlation value $(\geq 0.9)$ with azadirachtin 429 (Supplementary Table 8a) to perform quantitative PCR (qPCR). For this analysis, we used RNA from 
430 leaf, callus and developing seed (S1, S2, S3, S4 different stages of seed development) from neem

431 Genotype 1. This analysis showed that 8 out of 10 genes (Figure 6a) have high expression in 432 developing seeds and low expression in other tissues (leaf and callus). The qPCR results concordance 433 with WGCNA based clustering of RNA-seq and UHPLC-MS/SRM dataset. The highly expressed 434 genes such as transketolases (Ai02g19151 and Ai02g23582) and dehydrogenases (Ai02g25309 and 435 Ai02g12737) (Figure 6a) were among the top ranked in Pearson's co-relation value in WGCNA and 436 qPCR analysis.

\section{Organelle genomes assembly and annotation}

439 We filtered reads which map to chloroplast and mitochondrial genomes of other plant species. The 440 assembly and annotation statistics for organelle genome are shown in Table 4. The chloroplast genome 441 assembly contains 60 scaffolds with size of 112958 nts, which accounted for $72 \%$ of average plant 442 chloroplast genome. The chloroplast genome had N50 of 2,125 nts, GC content of $38.07 \%$ and longest 443

445 (Supplementary Table 18).

446 The mitochondrial genome of neem was also assembled (Table 4). N50 for mitochondrial genome had $4471490 \mathrm{nts}$, the sequence covered $266430 \mathrm{nts}$ of the genome in 348 scaffolds. The GC content of the 448 mitochondrial genome was $43.10 \%$. Gene prediction and annotation of the mitochondrial assembly was 449 done using Mitofy (Alverson et al. 2010), which identified 39 genes out of 41 reported mitochondrial 450 genes (Supplementary Table 19).

\section{Genome orthology and synteny analysis}

Genome size and chromosomal architecture was not available for neem tree. Synteny analysis was performed to obtain conserved chromosomal blocks of neem genome and genes in a closely related plant species. Our analysis revealed that neem genome is phylogentically related to citrus. Therefore, we used citrus genome (nine citrus pseudomolecules) as a reference to order neem contigs. This 
457 comparative analysis anchored 24,902 neem contigs onto 9 citrus chromosomes (Figure 7). This 458 anchoring method ordered $161 \mathrm{Mb}(62 \%)$ of neem genome covering, which is equivalent to $48 \%$ of 459 citrus genome. Detailed anchoring analysis revealed that 497 syntenic blocks with 12,176 synteny hit 460 with citrus genome (Figure 7).

461

462 Discussion

463 Neem is medicinally, agriculturally and environmentally important tropical tree in Indian subcontinent. 464 Neem is well-known for its complex tetranortriterpenoids compounds such as azadirachtin, nimbin and 465 salanin, which are the main constituents in insecticidal and pharmaceutical formulations (Brahmachari 466 2004). Unlike inorganic synthetic pesticides, neem compounds are best-known bio-pesticides, which 467 can easily be degraded and have lower pesticidal toxicity in the environmental. Biochemical 468 compounds from neem tree have been well investigated in the 20th century (Brahmachari 2004). 469 However, genetic, molecular and genomic resources are not well developed to understand genes and 470 biochemical pathways in neem. Recently, attempts were made to generate ESTs (Rajakani et al. 2014; 471 Narnoliya et al. 2014) and genomic (Krishnan et al. 2012) resources. However, these studies have 472 generated limited number of ESTs (Rajakani et al. 2014; Narnoliya et al. 2014) and also non473 availability of genome assembly and genes (Krishnan et al. 2012). Our study aimed to develop 474 comprehensive publicly available genomic, transcriptomic and metabolomic resources for neem tree.

475 We sequenced three independent neem genomes from three distinct regions of southern India. We 476 annotated the neem genome to the best of our knowledge using available bioinformatics tools. We 477 could able to assemble $70 \%(267 \mathrm{Mb})$ of genome based on estimated genome neem size by Ohri et al 478 (2004). More than 90\% of conserved eukaryotic genes (CEGs) mapped to de novo assembled neem 479 genome. Our prediction of genes $(44,495)$ and TE-related genes $(35 \%)$ are comparable with well 480 annotated rice genes (http://rice.plantbiology.msu.edu) (Kawahara et al. 2013). More than 30,000 genes 481 in the neem genome are supported by expression evidences, 13,711 genes expressed in most of neem 482 tissues and 3,000 genes expressed in a tissue specific manner. 
483 Our comprehensive analysis predicted about $87 \mathrm{Mb}(33 \%)$ repeats in the neem genome in contrast to 484 previous study (Krishnan et al. 2012). They have reported that neem genome contains the lowest repeat 485 content (13.03\%) in the plant kingdom (Krishnan et al. 2012). The previously published neem genome 486 by Krishnan et al has not released genome assembly and hence we cannot really make use of 487 information. We tried to re-built the neem genome using Krishnan et al short reads dataset 488 (SRA:1085705). This analysis predicted more than $20 \%$ of repeats and constitutes higher gaps with lots 489 of Ns (up to 60\%) (Supplementary Table 5). According to our knowledge, they have underestimated 490 the repeat content in the neem genome and their results are not reproducible (Supplementary Table 5). 491 Our study assembly generated from neem Genotype 1 showed higher repeats (33\%) content and lesser 492 Ns (less than 2\%) than Krishnan et al study.

493 Other highlight of our study is that we sequenced three neem genotypes from varied environmental 494 conditions, which assisted us to identify DNA molecular markers such as SSR, SNP and InDels. We 495 obtained about 2.9 SNPs and 0.22 InDels per 1000 nts on the reference neem genome (Genotype 1). 496 SNP and InDel markers density distribution in neem genome are lower than citrus genome (3.6 SNPs 497 and 0.6 InDels per Kb) (Xu et al. 2012). The lower SNP and InDel diversity in the neem genome and 498 the genic region indicates that neem trees might be genetically less diverse. Neem trees might be 499 forced to self pollinate because of bisexual nature of flowers, closed floral anatomy and lack of self 500 incompatibility (Puri et al. 1999). Other reason might be due to the presence of insect repellent 501 compounds in leaves and flowers, which may significantly reduce cross pollination. Molecular markers 502 (SNPs, InDels and SSRs) from this study are highly useful in identification of elite genotypes, tagging 503 of traits and cloning of genes involved in biochemical pathways in neem through genome-wide 504 association studies.

505 In the molecular phylogeny, neem is genetically related to sweet orange (Citrus sinensis) at the family 506 level (Xu et al. 2012). A lot more genetic and genomic resources are available for citrus plant (Wu et 507 al. 2014) as compared to citrus. Therefore we used citrus for comparative analysis with neem genome 508 (Figure $5 \mathrm{a})$. We observed that extensive syntenic blocks (62\% of neem genome) between neem and 509 citrus chromosomes and about 50\% $(24,216)$ of neem genes are conserved in the citrus genome. Citrus 
510 has been well researched plant for limonoids (Xu et al. 2012; Wu et al. 2014), which are highly 511 oxygenated limonoids present in both Rutaceae and Meliaceae. Molecular resources from our study 512 will help in dissecting common and specific limonoid pathways in neem and citrus.

513 The proposed biosynthetic pathway of azadirachtin in neem is not studied. The tirucallol (C30 514 triterpene) a steroid triterpenoid, is a possible precursor for azadirachtin biosynthesis in neem (Johnson 515 et al. 1996; Ley et al. 1993). In the first step, two molecules of farnesyl diphosphate combine to 516 generate tirucallol molecule followed by losing three methyl groups and oxidized to form apotirucallol 517 (a tetranortriterpenoid, or limonoid). Then it loses the four terminal carbons (Dewick 2011; Ley et al. 518 1993). The third ring of apotirucallol is oxidized to form the C-seco limonoids, nimbin and salannin 519 (Johnson et al. 1996; Ley et al. 1993; Puri 2004; Saxena 1989). These molecules are heavily oxidized 520 and cyclised to produce azadirachtin (Aerts \& Mordue 1997; Hosfelt 2008).

521 Our study added genomic, transcriptomic and metabolites data to support limonoids biosynthesis 522 pathway genes in neem. To understand the neem metabolites bio-synthesis, we quantified major 523 metabolites using a sensitive UHPLC-MS/SRM method. We identified the known secondary metabolite pathway genes including farnesyl diphosphate synthase (Ai02g10209), squalene synthase (Ai02g1634), geranyl diphosphate synthase (Ai02g31423), mevalonate kinase (Ai02g16520) and other unannotated genes upstream to squalene (Supplementary Table 20). Across different stages of developing seed (10 to 40 days after seed setting), we have observed increased content of major metabolites as compared to leaf and callus tissues (Figure 6b). In mature seeds, the concentration of neem metabolites [azadiractin $(11046 \mathrm{pg} / \mu \mathrm{g})$, nimbin $(3607 \mathrm{pg} / \mu \mathrm{g})$ and salanin $(5235 \mathrm{pg} / \mu \mathrm{g})$ ] was always higher than other neem tissues as reported earlier. Azadirachtin was 5000 fold higher in seed as compared to leaves. Azadirachtin is the major complex limonoids in neem tree. Genes involved in various steps from tirucallol to azadirachtin are not yet established. Genes identified from this study will help to identify biochemical pathways in neem. Clustering of genes expression and metabolites data was performed using WGCNA method, which aided in identification of genes that associate with azadirachtin biosynthesis pathways. The comprehensive resources from our study will help to unlock the biochemical pathways in neem. 


\section{CONCLUSION}

539 Neem is an important tropical evergreen tree in India, used for centuries in agriculture and traditional 540 medicine. In this study, we report the detailed analysis of neem genomes, transcriptomes and 541 metabolites. We identified possible candidate genes involved in azadirachtin biosynthesis pathways.

542 Genomic resources such as sequence of genomes, genes, transcripts, SSRs, SNPs and InDels from this 543 study will have a profound application to study diversity, traits associated with biopesticidal properties 544 and biochemical pathways in neem and other species of Melieacea family.

\section{Data access}

547 The genome assembly obtained from Illumina data of all the Genotypes has been submitted to NCBI 548 with accession number AMWY00000000.1 (Bioproject ID: PRJNA 176672) and raw reads deposited 549 in sequence read archive database (SRP052002).

\section{Acknowledgements}

552 This work is supported by Department of Biotechnology, Government of India to Malali Gowda 553 (Ramalingaswami Fellowship Grant; BT/HRD/35/02/2006). We acknowledge Genomics facility 554 (BT/PR3481/INF/22/140/2011) at Centre for Cellular and Molecular Platforms, Bangalore for 555 sequencing of Neem genomes. We acknowledge Pradeep H, Aarati Karaba, Manojkumar S and 556 Annapurna for their help in NGS library preparation and sequencing. We thank Ashmita G and Divya $557 \mathrm{~S}$ for their help in manual curation of SSR markers. We are grateful to Dr. Rajanna, National Botanical 558 Garden, University of Agricultural Science, GKVK campus, Bangalore for his help during neem 559 sample collection.

\section{References}

562 Aboyoun P, Pages H, and Lawrence M. 2010. GenomicRanges: Representation and manipulation of 563 genomic intervals. $R$ package version 1:1-25. 
564

565

566

567

568

569

570

571

572

573

574

575

576

577

578

579

580

581

582

583

584

585

586

587

588

589

590

591

592

593

594

595

596

597

598

Aerts RJ, and Mordue AJ. 1997. Feeding deterrence and toxicity of neem triterpenoids. Journal of Chemical Ecology 23:2117-2132.

Altschul SF, Gish W, Miller W, Myers EW, and Lipman DJ. 1990. Basic local alignment search tool. Journal of Molecular Biology 215:403-410.

Alverson AJ, Wei X, Rice DW, Stern DB, Barry K, and Palmer JD. 2010. Insights into the evolution of mitochondrial genome size from complete sequences of Citrullus lanatus and Cucurbita pepo (Cucurbitaceae). Molecular Biology and Evolution 27:1436-1448.

Boontong C, Pandey M, and Changtragoon S. 2009. Isolation and characterization of microsatellite markers in Indian neem (Azadirachta indica var. indica A. Juss) and cross-amplification in Thai neem (A. indica var. siamensis Valenton). Conservation Genetics 10:669-671.

Brahmachari G. 2004. Neem-an omnipotent plant: a retrospection. Chembiochem 5:408-421.

Broughton HB, Ley SV, Slawin AMZ, and Williams DJJ. 1986. X-Ray Crystallographic Structure of Detigloydihydroazadirachtin and Reassignment of the Structure of the Liminoid Insect Antifeedant Azadirachtin. Chem Soc, Chem Commun 1.

Burge C, and Karlin S. 1997. Prediction of complete gene structures in human genomic DNA. Journal of Molecular Biology 268:78-94.

Butterworth JH, and Morgan EDJ. 1968. Isolation of a substance that suppresses feeding in locusts. ChemCommun 1:52.

Chevreux B. 2005. MIRA: an automated genome and EST assembler. Ruprecht-Karls University, Heidelberg, Germany.

Cingolani P, Platts A, Coon M, Nguyen T, Wang L, Land SJ, Lu X, and Ruden DM. 2012. A program for annotating and predicting the effects of single nucleotide polymorphisms, SnpEff: SNPs in the genome of Drosophila melanogaster strain w1118; iso-2; iso-3. Fly 6:80-92.

Csüös M. 2010. Count: evolutionary analysis of phylogenetic profiles with parsimony and likelihood. Bioinformatics 26:1910-1912.

Delcher AL, Harmon D, Kasif S, White O, and Salzberg SL. 1999. Improved microbial gene identification with GLIMMER. Nucleic Acids Research 27:4636-4641.

Dewick PM. 2011. Medicinal natural products: a biosynthetic approach: John Wiley \& Sons.

Doyle JJ. 1990. Isolation of plant DNA from fresh tissue. Focus 12:13-15.

Drożdżyński D, and Kowalska J. 2009. Rapid analysis of organic farming insecticides in soil and produce using ultra-performance liquid chromatography/tandem mass spectrometry. Analytical and Bioanalytical Chemistry 394:2241-2247.

Grabherr MG, Haas BJ, Yassour M, Levin JZ, Thompson DA, Amit I, Adiconis X, Fan L, Raychowdhury R, and Zeng Q. 2011. Full-length transcriptome assembly from RNA-Seq data without a reference genome. Nature Biotechnology 29:644-652. 
Grimalt S, Thompson DG, Coppens M, Chartrand DT, Shorney T, Meating J, and Scarr T. 2011. Analytical Study of Azadirachtin and 3-Tigloylazadirachtol Residues in Foliage and Phloem of Hardwood Tree Species by Liquid Chromatography-Electrospray Mass Spectrometry. Journal of Agricultural and Food Chemistry 59:8070-8077.

Heasley B. 2011. Synthesis of limonoid natural products. European Journal of Organic Chemistry 2011:19-46.

Hosfelt J. 2008. Azadirachtin. Chemistry 150.

Jiang H, and Wong WH. 2008. SeqMap: mapping massive amount of oligonucleotides to the genome. Bioinformatics 24:2395-2396.

Jiang H, and Wong WH. 2009. Statistical inferences for isoform expression in RNA-Seq. Bioinformatics 25:1026-1032.

Johnson S, Morgan ED, and Peiris CN. 1996. Development of the Major Triterpenoids and Oil in the Fruit and Seeds of Neem (Azadirachta indica). Annals of Botany 78:383-388.

Kawahara Y, de la Bastide M, Hamilton JP, Kanamori H, McCombie WR, Ouyang S, Schwartz DC, Tanaka T, Wu J, and Zhou S. 2013. Improvement of the Oryza sativa Nipponbare reference genome using next generation sequence and optical map data. Rice 6:4.

Kent WJ. 2002. BLAT - the BLAST-like alignment tool. Genome Research 12:656-664.

Krishnan N, Pattnaik S, Jain P, Gaur P, Choudhary R, Vaidyanathan S, Deepak S, Hariharan A, Krishna PG, and Nair J. 2012. A draft of the genome and four transcriptomes of a medicinal and pesticidal angiosperm Azadirachta indica. BMC Genomics 13:464.

Kurtz S, Phillippy A, Delcher A, Smoot M, Shumway M, Antonescu C, and Salzberg S. 2004. Versatile and open software for comparing large genomes. Genome Biology 5.

Langfelder P, and Horvath S. 2008. WGCNA: an R package for weighted correlation network analysis. BMC Bioinformatics 9:559.

Langmead B, and Salzberg SL. 2012. Fast gapped-read alignment with Bowtie 2. Nature Methods 9:357-359.

Lechner M, Findeĩ̃̃̈ S, Steiner L, Marz M, Stadler PF, and Prohaska SJ. 2011. Proteinortho: Detection of (Co-) orthologs in large-scale analysis. BMC Bioinformatics 12:124.

Ley SV, Denholm AA, and Wood A. 1993. The chemistry of azadirachtin. Nat Prod Rep 10:109-157.

Li H, Handsaker B, Wysoker A, Fennell T, Ruan J, Homer N, Marth G, Abecasis G, Durbin R, and Genome Project Data Processing S. 2009. The Sequence Alignment/Map format and SAMtools. Bioinformatics (Oxford, England) 25:2078-2079.

Li W, and Godzik A. 2006. CD-hit: a fast program for clustering and comparing large sets of protein or nucleotide sequences. Bioinformatics 22:1658-1659.

Metzker ML. 2010. Sequencing technologies - the next generation. Nature Reviews Genetics 11:31-46. 
634 Ming R, VanBuren R, Liu Y, Yang M, Han Y, Li L-T, Zhang Q, Kim M-J, Schatz MC, and Campbell

635

636

637

638

639

640

641

642

643

644

645

646

647

648

649

650

651

652

653

654

655

656

657

658

659

660

661

662

663

664

665

666

667

668

M. 2013. Genome of the long-living sacred lotus (Nelumbo nucifera Gaertn.). Genome Biology 14:R41.

Narnoliya LK1, Rajakani R, Sangwan NS, Gupta V and Sangwan RS. 2014. Comparative transcripts profiling of fruit mesocarp and endocarp relevant to secondary metabolism by suppression subtractive hybridization in Azadirachta indica (neem). Molecular Biology Reports 41(5):R41.

Ohri D, Bhargava A, and Chatterjee A. 2004. Nuclear DNA Amounts in 112 Species of Tropical Hardwoods-New Estimates. Plant Biology 6:555-561.

Parra G, Bradnam K, and Korf I. 2007. CEGMA: a pipeline to accurately annotate core genes in eukaryotic genomes. Bioinformatics 23:1061-1067.

Puri HS. 2004. Neem: The divine tree Azadirachta indica: CRC Press.

Puri HS. 1999. Neem, The divine tree, Azadirachta indica 182 pp. Harwood Academic Publishers, The Netherlands.

Rajakani R, Narnoliya L, Sangwan NS, Sangwan RS, and Gupta V. 2014. Subtractive transcriptomes of fruit and leaf reveal differential representation of transcripts in Azadirachta indica. Tree Genetics \& Genomes 10:1331-1351.

Ray S, and Satya P. 2014. Next generation sequencing technologies for next generation plant breeding. Frontiers in Plant Science 5.

Saxena RC. 1989. Insecticides from neem. Insecticides of Plant Origin 387:110-135.

Schmid R, and Blaxter ML. 2008. annot8r: GO, EC and KEGG annotation of EST datasets. BMC Bioinformatics 9:180.

Siddiqui S. 1942. A note on the isolation of three new bitter principles from the nim oil. Current Science 11:278-279.

Sidhu O, Kumar V, and Behl H. 2003. Variability in Neem (Azadirachta indica) with respect to azadirachtin content. Journal of Agricultural and Food Chemistry 51:910-915.

Smit A, and Hubley R. 2008. RepeatModeler Open-1.0. 2008-2010.

Soderlund C, Bomhoff M, and Nelson WM. 2011. SyMAP v3. 4: a turnkey synteny system with application to plant genomes. Nucleic Acids Research 39:e68-e68.

Stanke M, Keller O, Gunduz I, Hayes A, Waack S, and Morgenstern B. 2006. AUGUSTUS: ab initio prediction of alternative transcripts. Nucleic Acids Research 34:W435-W439.

Tan Q-G, and Luo X-D. 2011. Meliaceous limonoids: chemistry and biological activities. Chemical Reviews 111:7437-7522.

Varshney RK, Chen W, Li Y, Bharti AK, Saxena RK, Schlueter JA, Donoghue MT, Azam S, Fan G, and Whaley AM. 2012. Draft genome sequence of pigeonpea (Cajanus cajan), an orphan legume crop of resource-poor farmers. Nature Biotechnology 30:83-89. 
669 Veitch GE, Boyer A, and Ley SV. 2008. The azadirachtin story. Angewandte Chemie International $670 \quad$ Edition 47:9402-9429.

671 Warnes GR, Bolker B, and Lumley T. 2008. gtools: Various R programming tools. $R$ package version 6722.

673 Wickham H. 2010. stringr: Make it easier to work with strings., 2010. URL http://CRAN R-project $674 \quad$ org/package $=$ stringr $R$ package version 04 .

675 Weizhong Li \& Adam Godzik. 2006. Cd-hit: a fast program for clustering and comparing large sets of 676 protein or nucleotide sequences. Bioinformatics 22:1658-9.

677 Wyman SK, Jansen RK, and Boore JL. 2004. Automatic annotation of organellar genomes with 678 DOGMA. Bioinformatics 20:3252-3255.

679 Xu Q, Chen L-L, Ruan X, Chen D, Zhu A, Chen C, Bertrand D, Jiao W-B, Hao B-H, and Lyon MP. 680 2013. The draft genome of sweet orange (Citrus sinensis). Nature Genetics 45:59-66.

681 Zerbino DR, and Birney E. 2008. Velvet: algorithms for de novo short read assembly using de Bruijn 682 683 684 685 graphs. Genome Research 18:821-829.

Zhang X, and Wessler SR. 2004. Genome-wide comparative analysis of the transposable elements in the related species Arabidopsis thaliana and Brassica oleracea. Proceedings of the National Academy of Sciences of the United States of America 101:5589-5594.

686

687 
Estimation of major neem metabolites from different tissues of neem

(A) Neem tree, (B) structure of neem metabolites, (C) UHPLC-MS/SRM chromatogram of standards and metabolites from seed extract, (D) standard curve for all three metabolites and $(E)$ concentration of neem metabolites from various tissues of neem tree.

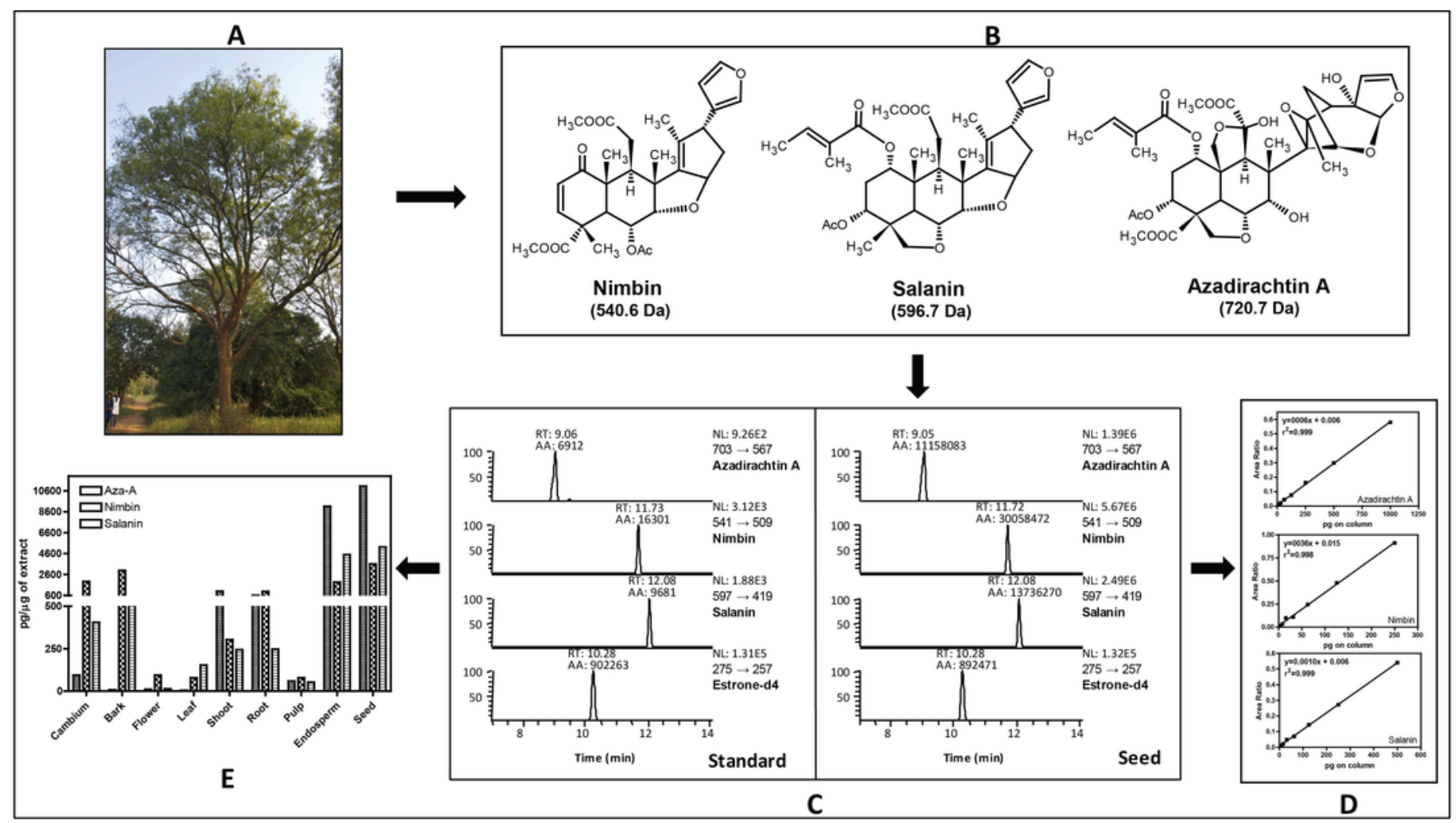


Figure 2 (on next page)

Gene expression and metabolite differences among different tissues of neem

(A) Comparison of genes expressed commonly and uniquely in flower and bud, developing endosperm, mature leaf, mature fruit, fruit coat and pulp tissues. (b) Clustering dendrogram of samples based on gene expression values and corresponding metabolite concentration. The heat map shows the higher expression of the genes in specific tissue 
A

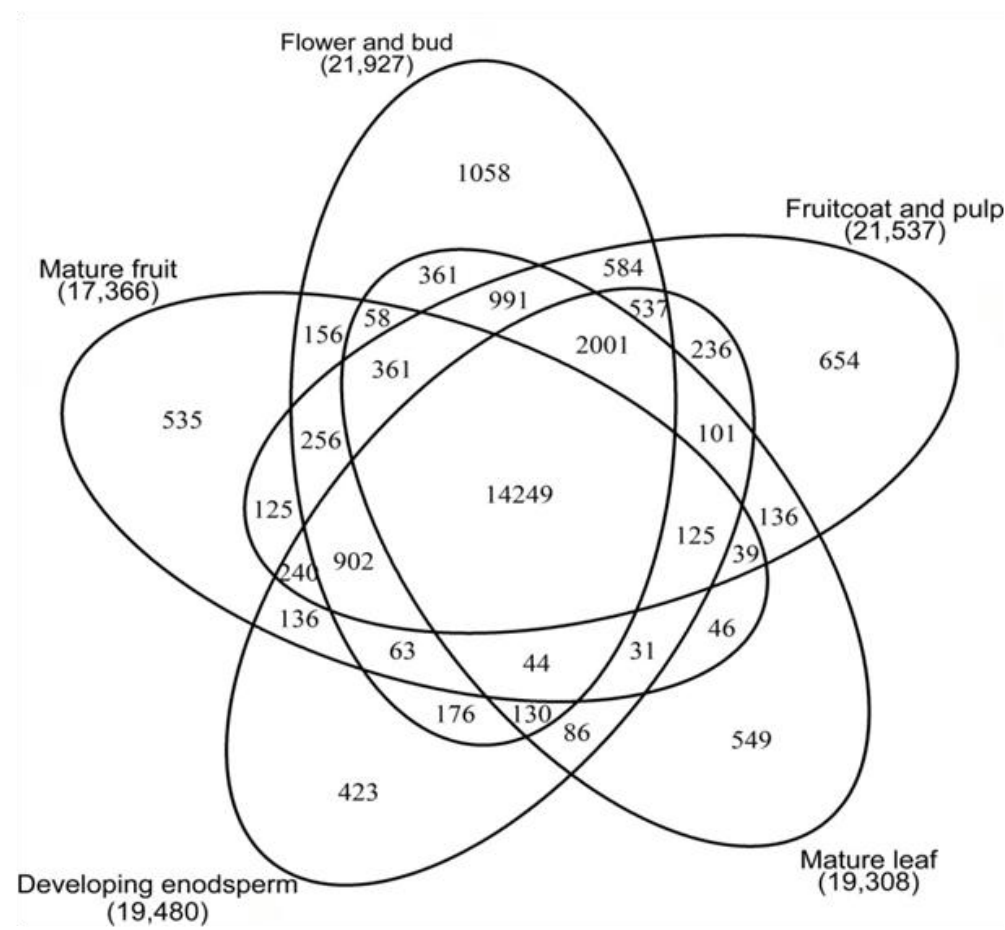

B

Tissue dendrogram based on gene expression and trait heatmap
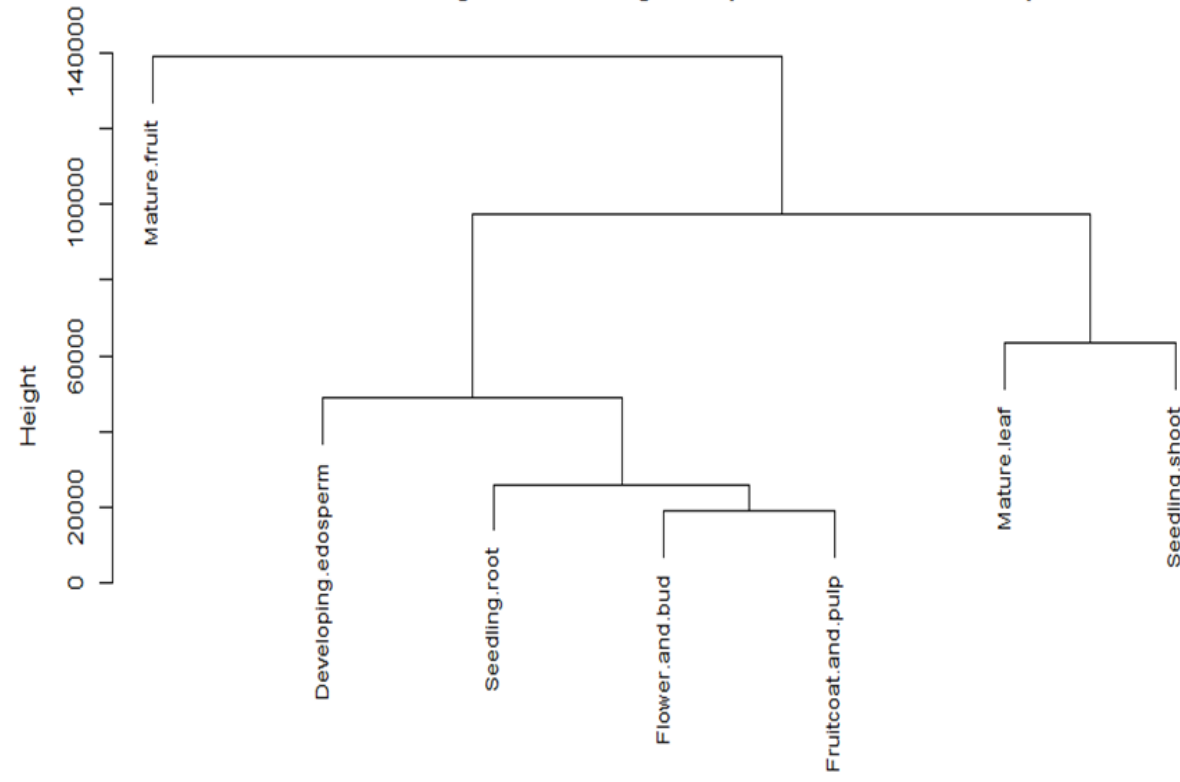

Azadirachtin

Nimbin

Salanin

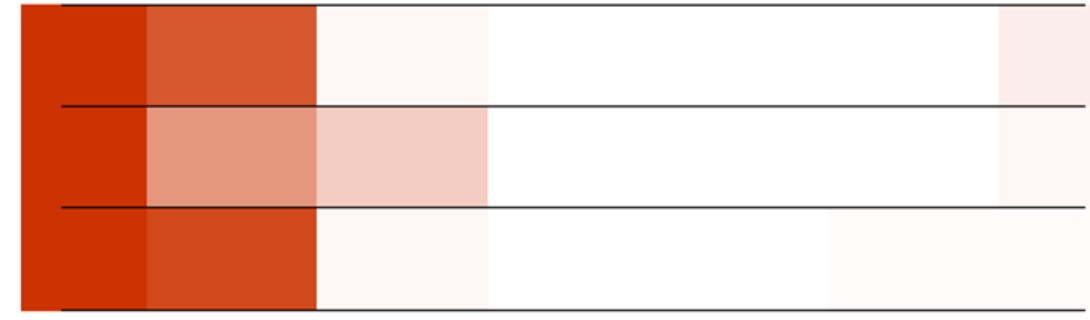




\section{Figure $\mathbf{3}$ (on next page)}

Proposed Azadirachtin biosynthetic pathway in A. indica

Azadirachtin is the most prominent biopestiside belonging to organic molecule class called tetranortriterpenoids. Triterpenoids derived basically from squalene which in turn is derived from geranyl

pyrophosphate (GPP). GPP can be derived from mevalonate (MVA) or 2-C-methyl-D875 erythritol 4-phosphate (MEP) pathway. Enzymes of MVA pathway are as follows:

AACT =acetyl-CoA acetyltransferase; HMGS=3-hydroxy-3-methylglutaryl CoA synthase; $\mathrm{HMGR}=3$-hydroxy-3-methylglutaryl-coenzyme $\mathrm{A}$ reductase; $\mathrm{MVK}=$ mevalonate kinase; $P M K=$ phosphomevalonate kinase; $P M D=$ diphosphomevalonate decarboxylase. Enzymes of MEP pathway are DXS=1-deoxy-D-xylulose-5-phosphate synthase; DXR=1deoxy-D xylulose-5-phosphate reducto-isomerase; $M C T=2-C$-methyl-D-erythritol 4-phosphate cytidyly|transferase; CMK=4-diphosphocytidyl-2-C-methyl-D-erythritol kinase; MDS=2-C methyl-D-erythritol 2,4-cyclodiphosphate synthase; HDS=4-hydroxy-3-methylbut-2-enyl diphosphate synthase; HDR=4-hydroxy-3-methylbut-2-enyl diphosphate reductase. Isopentenyl pyrophosphate isomerase (IPPI) catalyzes the isomerisation of isopentenyl pyrophosphate (IPP) to dimethylallyl pyrophosphate (DMAPP), whereas conversion of IPP to geranyl pyrophosphate (GPP) is catalyzed by geranyl pyrophosphate synthase (GPS). GPP is further converted to farnesyl-diphosphate (FPP) and squalene by farnesyl-diphosphate synthase (FPS) and squalene synthase (SS) respectively. Solid arrows indicate known steps, whereas broken arrows represents unknown intermediates and enzymes. Numbers beside coloured blocks indicate tissue types $(1=$ developing endosperm; $2=$ mature leaf; $3=$ mature fruit; $4=$ seedling root; $5=$ fruit coat and pulp; $6=$ seedling shoot; $7=$ open flower and flower bud) and heatmap is represented as expression RPKM value. 


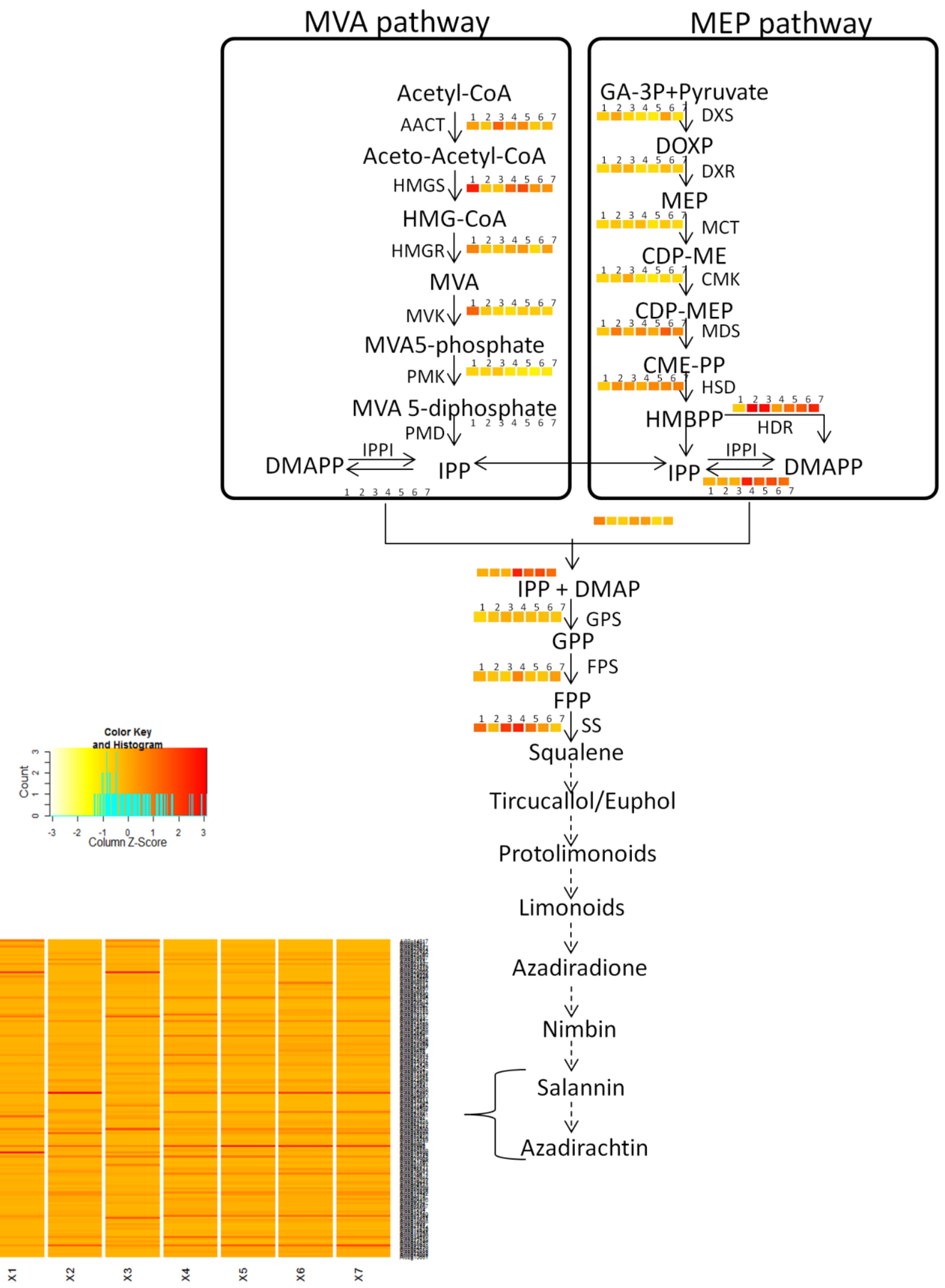


Figure 4 (on next page)

Co-orthologous groups detected by Proteinortho using UPGMA method.

The clustering is based on similarity of the proteome among the species. 


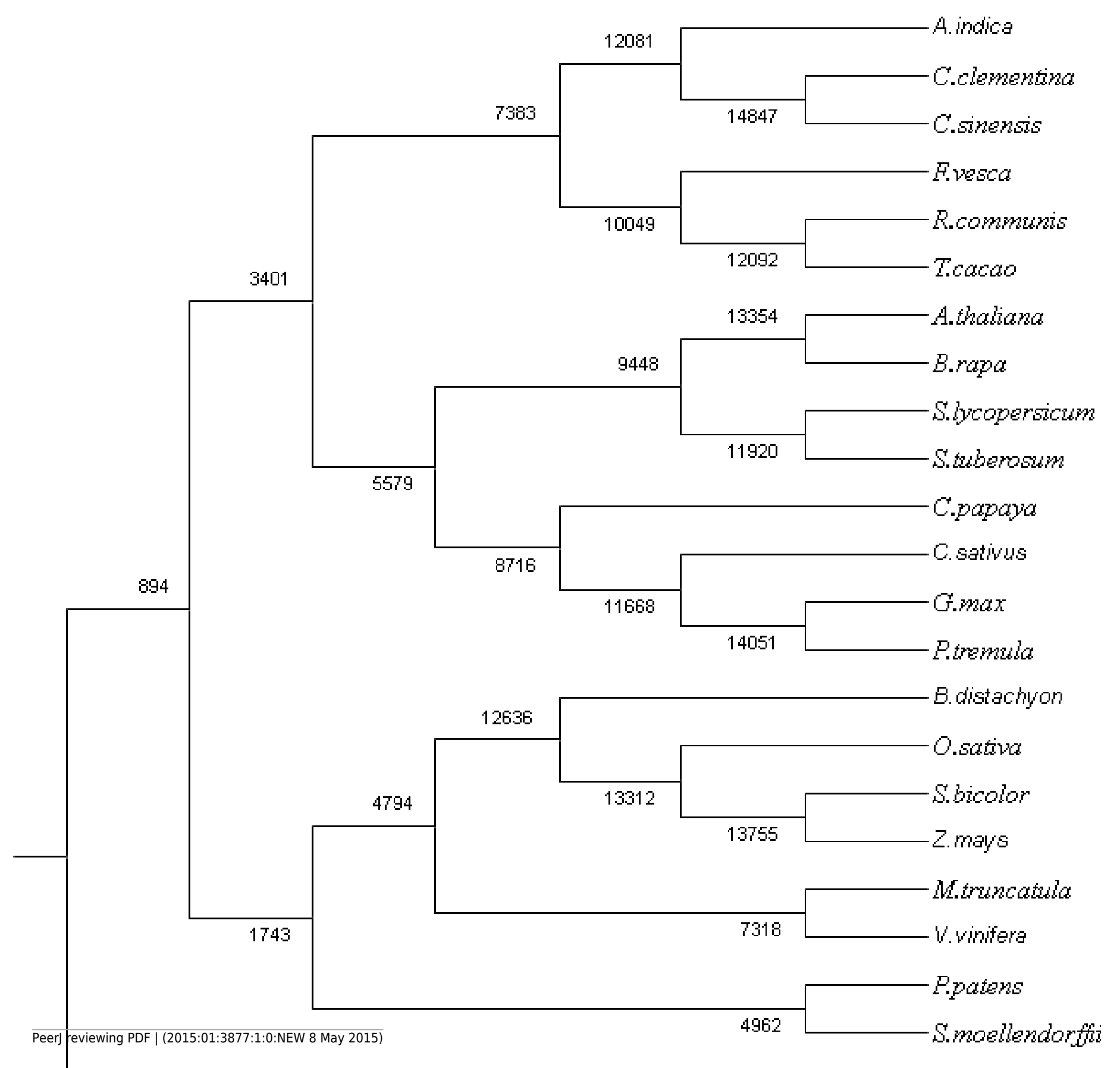




\section{5}

Comparison of neem genes with other sequenced plants.

(A) Schematic diagram showing common and unique genes between citrus and neem genomes. (B) number and percentage of proteins having homolog hit in query (Arabidopsis, Populus, grapes, caster and rice) with greater than $60 \%$ identity in genomes of neem and citrus.

A

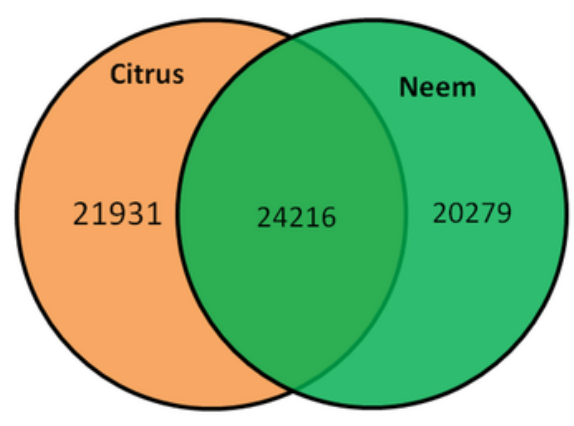

B

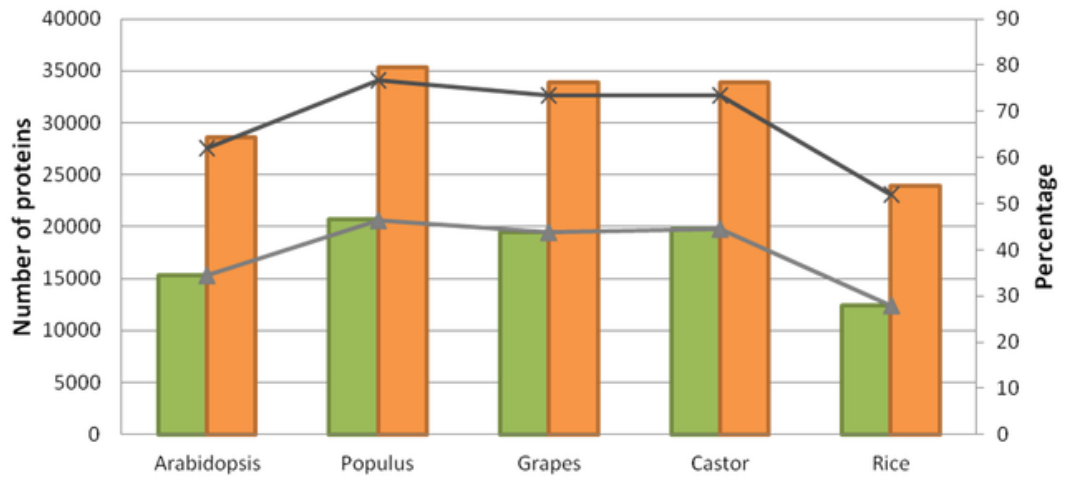




\section{6}

Correlation of gene expression using qPCR and metabolites content in various neem tissues.

(A) qPCR performed with genes selected from RNA-seq data. Developing seeds were selected across different stages (S1 - developing seed 1, S2 - developing seed 2, S3 - developing seed , 54 - developing seed) based on growth stages show high gene expression as compared to other tissues. (B) LC-MS quantified metabolites data from various tissues.

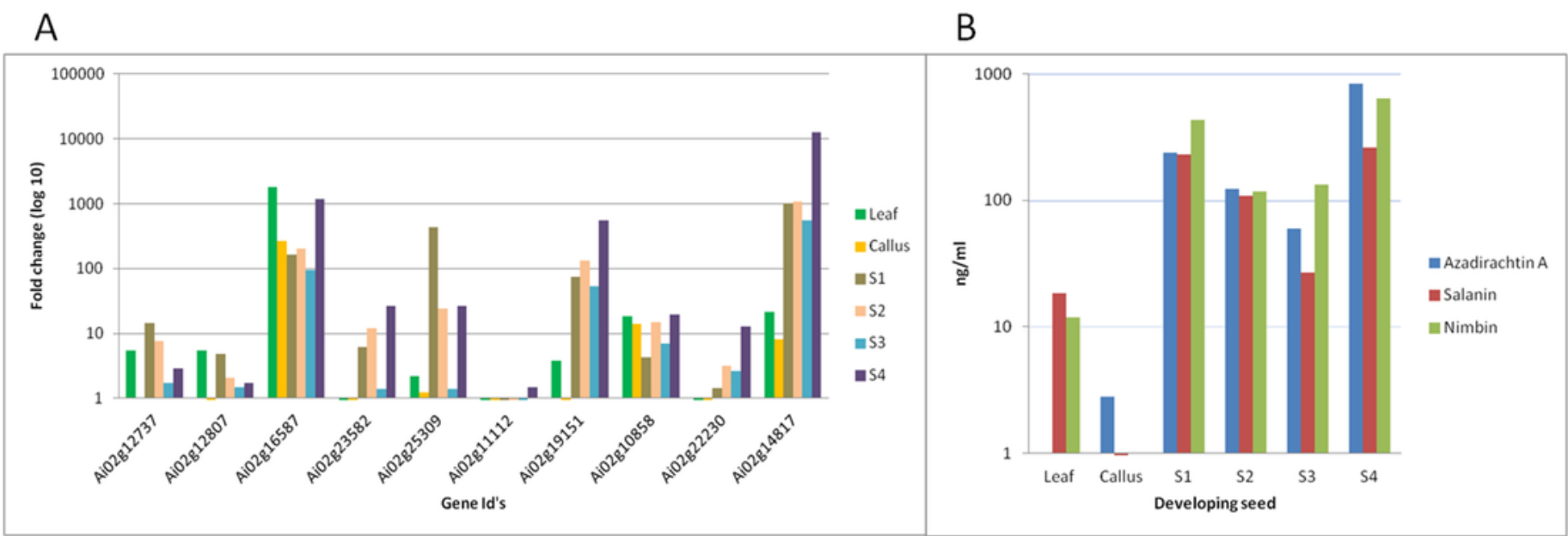




\section{7}

Schematic representation of syntenic relationship between citrus and neem.

The colored line (1-9, UNK) represents the syntenic blocks in neem anchored to chromosomal region in citrus. The Turquoise colored blocks show the synteny of un-anchored neem scaffolds with citrus.

Neem

Citrus

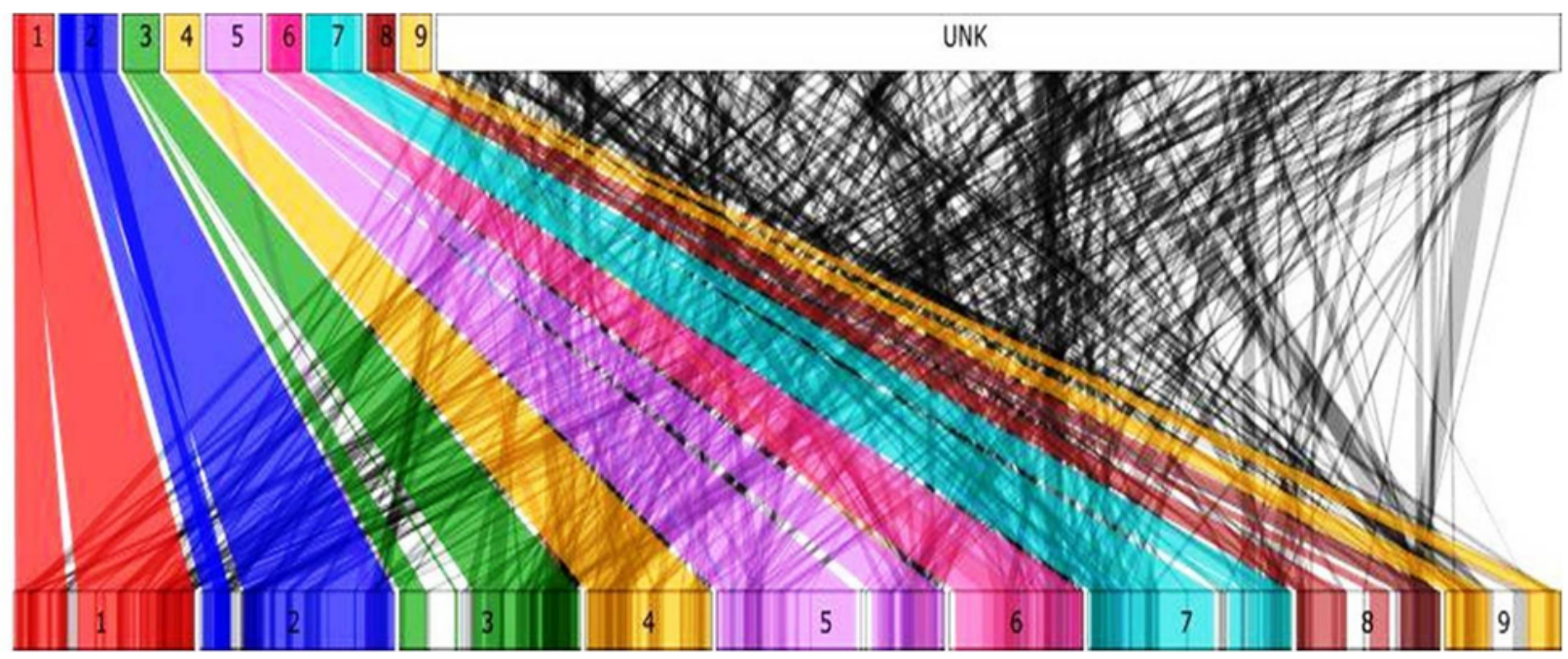




\section{Table $\mathbf{1}$ (on next page)}

Neem genome assembly statistics 


\begin{tabular}{llll}
\hline & $\begin{array}{l}\text { Velvet } \\
\text { assembly from } \\
\text { Illumina reads }\end{array}$ & $\begin{array}{l}\text { MIRA assembly } \\
\text { from 454 reads }\end{array}$ & $\begin{array}{l}\text { Hybrid assembly } \\
\text { of Genotype 1 }\end{array}$ \\
\hline Total high quality reads & 168895379 & 2762254 & - \\
k-mer & 45 & - & - \\
Assembled genome size (Mb) & 216 & 157 & 268 \\
Total number of contigs & 94,780 & $1,21,184$ & 68,604 \\
N50 (bp) & 22,263 & 1463 & 15,948 \\
Maximum contig length (bp) & $2,41,126$ & 43859 & 241170 \\
Mininum contig length (bp) & 89 & 52 & 89 \\
\% of bases in contigs $\geq 1000 b p$ & 93.31 & 74.54 & 94.65 \\
Total repeat size in Mb (\%) & $59.81(27.41)$ & $48.84(31.05)$ & $86.90(32.44)$ \\
Number of predicted genes in Augustus & 27,556 & 41,169 & 40,130 \\
Number of predicted genes in GeneScan & 35,501 & 57,356 & 52,617 \\
Number of Genes clustered from GeneScan & & & 48,032 \\
and Augustus & 37,161 & 61,901 & \\
No of genes with $>100$ bp & 34,992 & 52,957 & 44,495 \\
Genes with RNA seq evidence & 27,087 & 43,383 & 32,278 \\
Non -TE genes & 19,547 & 41,373 & 29,050 \\
\hline
\end{tabular}




\section{Table 2 (on next page)}

RNAseq analysis from various explants of neem

$*$ read quality $=q 20$ and read length $=100$ nts 


\begin{tabular}{|c|c|c|c|c|}
\hline Tissue & No. of reads* & $\begin{array}{l}\text { No of } \\
\text { genes/RPKM }>1\end{array}$ & $\begin{array}{l}\text { No of } \\
\text { genes/RPKM > } \\
5\end{array}$ & $\begin{array}{ll}\text { No } & \text { of } \\
\text { genes/RPKM } \\
10\end{array}$ \\
\hline Mature leaf & 5401910 & 19308 & 14807 & 11763 \\
\hline Flower and bud & 22654982 & 21927 & 16716 & 13632 \\
\hline Fruit coat and pulp & 55627021 & 21537 & 16693 & 13888 \\
\hline $\begin{array}{l}\text { Developing } \\
\text { endosperm }\end{array}$ & 31340522 & 19480 & 15262 & 12614 \\
\hline Mature fruit & 23321657 & 17366 & 12407 & 9741 \\
\hline Seedling root & 4427659 & 20798 & 17015 & 14312 \\
\hline Seedling shoot & 5894621 & 20199 & 15926 & 13018 \\
\hline Drought root & 7255199 & 21371 & 17015 & 14236 \\
\hline Drought shoot & 22600138 & 20763 & 16077 & 13431 \\
\hline Albino root & 12674871 & 21710 & 17201 & 14273 \\
\hline Albino shoot & 23115676 & 21226 & 16874 & 14066 \\
\hline Leaf callus & 2150935 & 18615 & 15356 & 12727 \\
\hline
\end{tabular}


Table 3 (on next page)

De novo repeat prediction from neem genome using Repeat Modeler program 
2

\begin{tabular}{|c|c|c|c|c|}
\hline Repeat type & Subclass & $\begin{array}{l}\text { Number } \\
\text { of } \\
\text { elements }\end{array}$ & Length in bp (\%) & $\begin{array}{l}\% \text { of } \\
\text { sequences }\end{array}$ \\
\hline \multicolumn{5}{|l|}{ RNA elements: } \\
\hline SINEs: & & 119 & 10033 bp (0.01) & 0.00 \\
\hline \multirow[t]{3}{*}{ LINEs: } & & 2554 & $1240701 \mathrm{bp}(0.46)$ & 0.46 \\
\hline & LINE1 & 1918 & 1002661 bp (0.37) & 0.37 \\
\hline & LINE2 & 119 & $39194 \mathrm{bp}(0.01)$ & 0.01 \\
\hline \multirow[t]{2}{*}{ LTR elements: } & & 51260 & $26838058 \mathrm{bp}(10.02)$ & 10.02 \\
\hline & ERV class 1 & 462 & 130859 bp $(0.05)$ & 0.05 \\
\hline DNA elements: & & 17356 & 7112032 bp (2.65) & 2.65 \\
\hline Unclassified: & & 166584 & $45693338 \mathrm{bp}(17.06)$ & 17.06 \\
\hline $\begin{array}{l}\text { Total interspersed } \\
\text { repeats: }\end{array}$ & & & 80894162 bp (30.20) & 30.20 \\
\hline Simple repeats: & & 43430 & $1601548 \mathrm{bp}(0.60)$ & 0.60 \\
\hline Low complexity: & & 99976 & $5150192 \mathrm{bp}(1.92)$ & 1.92 \\
\hline
\end{tabular}




\section{Table 4(on next page)}

Mitochondria and chloroplast genomes assembly statistics 


\begin{tabular}{lll}
\hline Assembly parameters & Mitochondrion & Chloroplast \\
\hline Total number of reads & 15659391 & 22211576 \\
k-mer & 63 & 61 \\
Assembled genome size (bp) & 266430 & 112958 \\
Total number of contigs & 348 & 152 \\
N50 (bp) & 1490 & 2125 \\
Maximum contig length (bp) & 9110 & 8435 \\
Minimum contig length (bp) & 125 & 121 \\
\% of bases in contigs $\geq 1000 \mathrm{bp}$ & 60.71 & 63.33 \\
GC content \% & 43.1 & 38.07 \\
No of genes predicted & 39 & 77 \\
\hline
\end{tabular}

